\title{
Rigid Body Spring Network Model for Plasticity and Fracture
}

\author{
Christos D. Sofianos, Vlasis. K. Koumousis \\ National Technical University of Athens \\ Zografou Campus, 15780, Athens, Greece \\ sofianoschristos@yahoo.gr; vkoum@central.ntua.gr
}

\begin{abstract}
In this work the concept of the Rigid Body Spring Network model (RBSN) is extended to account for hysteresis and brittle fracture of structural members. RBSN was proposed by Kawai [1] who employed a simple discrete numerical model that expresses the phenomenological properties of the material based on rigid masses and springs that undergo all the structural deformation. Material is discretized based on Voronoi [2] tessellation into convex polygons that form the discrete rigid bodies of the model. These are interconnected with three zero length springs in the middle of their common facets. The behavior of springs follows the smooth hysteretic Bouc-Wen model [7]. Crack initiation and propagation is constrained at the rigid body facets. Random mesh generation, using only a minimum distance criterion is used and effectively minimizes the mesh bias towards crack propagation due to monotonic loading. Crack formation obeys simple cut-off and Mohr-Coulomb type of criteria [3]. Based on this formulation, the different states are closely followed and the plastic as well as fracture behavior is manifested for highly stressed regions. Numerical results are presented for 2D plane stress models that validate the proposed method and verify its computational efficiency as compared to standard elastoplastic finite element methods.
\end{abstract}

Keywords: Hysteresis, Fracture, RBSN, plasticity

\section{Introduction}

The concept of a Rigid Body Spring Network model (RBSN) was first proposed by Kawai [1]. The main idea is to divide the structure into discrete rigid bodies that are connected with springs. The aim is to reduce the computational resources needed for satisfactory simulation in comparison with other methods such as the Finite Element method, since the elemental stiffness matrices never exceed the 6x6 dimensions irrespective of beam, plate, or shell elements. Since then the RBSN model has been used widely especially in the simulation of structural concrete and the modelling of fracture [2], [3]. In their work Bolander and Saito [4] proposed the scaling of elemental stiffness according to Voronoi tessellation of the domain in order to ensure elastic uniformity and maximize the isotropy of crack path propagation, since crack movement is confined within the boundaries between particles and is therefore strongly affected by the implemented meshing technique. Their work was based on static loading, which was applied in increments in concrete members in order to investigate the crack formation and crack path. Kim and Lim [5] applied the idea of Voronoi scaling to simulate concrete fracture under dynamic loading using visco-plastic damage model to describe the rate dependency. In this work a rate independent plasticity model such as the Bouc-Wen model of hysteresis is applied to the Rigid Body Spring Network framework to simulate plasticity.

The Bouc-Wen hysteretic model has been widely utilized to express a range of hysteretic behavior, where a system's response depends not only on its current state but also on the history of its previous states. Hysteretic models incorporating stiffness degradation, strength deterioration, and pinching phenomena have been effective in simulating the real behavior of various materials such as reinforced concrete, steel and wood. The Bouc-Wen model introduces internal variables that are governed by nonlinear differential equations that are expressed in rate form at the element level. Efficient algorithms have been developed for the identification of the Bouc-Wen model parameters, using advanced analytical techniques or evolutionary identification approaches.

\section{The RBSN model}

In the RBSN model the structure is divided into a set of rigid bodies that are connected, in the middle of each of their common boundaries, with three zero length springs. Structure deformation is the result of the localized deformations of 
these internal springs. The rigid bodies or cells are assumed to be rigid meaning that they can't deform, but they can separate or even overlap. The displacements of the cells centroid $\left(u_{1}, v_{l}, \theta_{l}\right)$, are those that determine the motion of the cell. The displacement of any point inside the cell with coordinates $(x, y)$ is given in (1), where $\theta_{l}$ is a small angle:

$$
\begin{gathered}
u=u_{1}-\left(y-y_{1}\right) \theta_{1} \\
v=v_{1}-\left(x-x_{1}\right) \theta_{1}
\end{gathered}
$$

According to Bolander and Saito [4], for a two dimensional case, the maximum number of points that can be placed in a rectangular domain using a random distribution process is $m_{n}=0.68 a b / d_{m}{ }^{2}$ where $a$ and $b$ are the height and width of the rectangular domain and $d_{m}$ is a minimum allowable distance between points. As the number of points that form the seeds for the discretization process approach $m_{n}$ the mesh becomes more regular and the points tend to be more uniformly distributed. This formulation is necessary because the Voronoi scaling of spring constants ensures elastic uniformity, for simple modes of straining and reduces the bias of the mesh towards crack propagation.

In the first step a set of points approaching $m_{n}$ are randomly placed in the domain following the minimum distance criterion. These points form the nuclei of the Voronoi diagram [6]. The Voronoi diagram divides the domain into convex polygons i.e. the Voronoi cells that define the rigid particles of the model. Voronoi cell $I$ is defined as the set of points that are closer to its generator node $I$ than any other node in the system. Each individual element consists of two rigid particles and their common facet as shown in Figure 1, where, by the way the mesh is generated, the line connecting the computational points 1 and 2 will always be perpendicular to the common facet connecting points 3 and 4 .

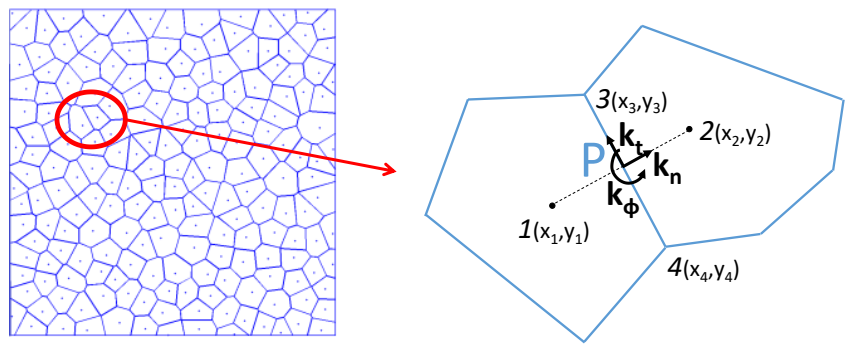

Fig. 1: Voronoi tessellation and Rigid Body Spring Network element.

The coordinates of the computational points for a two particle assembly are $\left(x_{1}, y_{1}\right)$ and $\left(x_{2}, y_{2}\right)$ and the coordinates of the edges of their common boundary are $\left(x_{3}, y_{3}\right)$ and $\left(x_{4}, y_{4}\right)$. At the middle of the interface (point $\left.\mathrm{P}\right)$ three zero length springs lie with stiffness $k_{n}, k_{t}$ and $k_{\varphi}$ in the normal, tangential and rotational local degrees of freedom. The diagonal matrix [D] of these uncoupled stiffnesses can be defined:

$$
[D]=\left[\begin{array}{ccc}
k_{n} & 0 & 0 \\
0 & k_{t} & 0 \\
0 & 0 & k_{\varphi}
\end{array}\right]
$$

The displacements at point $P$ in the facet local system are:

$$
\{d\}^{T}=\left[\begin{array}{lll}
\delta_{n} & \delta_{t} & \varphi
\end{array}\right]
$$

and the nodal displacements of the two reference nodes in the global system:

$$
\left\{u_{e}\right\}^{T}=\left[\begin{array}{llllll}
u_{1} & v_{1} & \theta_{1} & u_{2} & v_{2} & \theta_{2}
\end{array}\right]
$$

These are connected through the relation: 


$$
\{d\}=[B]\left\{u_{e}\right\}
$$

where $[B]$ matrix is a matrix derived from geometrical considerations Error! Reference source not found.:

$$
[B]=\frac{1}{l_{43}}\left[\begin{array}{cccccc}
y_{43} & -x_{43} & \left(-x_{43} x_{P 1}-y_{43} y_{P 1}\right) & -y_{43} & x_{43} & \left(x_{43} y_{P 2}+y_{43} y_{P 2}\right) \\
x_{43} & y_{43} & \left(y_{43} x_{P 1}-x_{43} y_{P 1}\right) & -x_{43} & -y_{43} & \left(-y_{43} x_{P 2}+x_{43} y_{P 2}\right) \\
0 & 0 & -l_{43} & 0 & 0 & l_{43}
\end{array}\right]
$$

where $x_{i j}=x_{i}-x_{j}, y_{i j}=y_{i}-y_{j}, l_{i j}$ is the Euclidean distance between points $i$ and $j, x_{p i}=\left(x_{4 i}+x_{3 i}\right) / 2, y_{p i}=\left(y_{4 i}+y_{3 i}\right) / 2$. In addition, the internal forces of the springs $\{q\}$ are evaluated with the use of the following relation:

$$
\{q\}=\left\{\begin{array}{c}
F_{n} \\
F_{t} \\
M
\end{array}\right\}=[D]\{d\}
$$

where $F_{n}, F_{t}$ are the spring forces in the normal and tangential direction respectively and $M$ is the moment of the rotational spring. In order to approximate the elastic properties of the continuum, spring stiffness is scaled based on the Voronoi discretization as:

$$
\begin{aligned}
& k_{n}=E^{\prime} t l_{43} / h \\
& k_{t}=E^{\prime \prime} t l_{43} / h \\
& k_{\varphi}=k_{n} l_{43}^{2} / 12
\end{aligned}
$$

where for plane stress conditions $E^{\prime}=E /\left(1-v^{2}\right)$ and $E^{\prime \prime}=E /(2(1+v)), E$ is the elastic modulus, $v$ the Poisson ratio, $t$ the thickness of the domain and $h$ the distance of the two nodes. In order to obtain the elemental stiffness matrix, the virtual work theorem is applied and the resulting stiffness matrix is:

$$
[K]_{e}=[B]^{T}[D][B]
$$

All elemental stiffness matrices are then assembled into the global stiffness matrix of the structure using the direct stiffness method. The final global stiffness matrix is square and has dimensions of $(3 \times n, 3 \times n)$ where $n$ is the number of nodes.

\section{The Bouc-Wen model}

In this work the Bouc-Wen hysteretic model is employed to account for plasticity related phenomena. It is a smooth hysteretic model that was first proposed by Bouc [7] and was later extended by Wen [8]. The model is capable of capturing a broad range of hysteretic behaviors and is based on differential equations. Its mathematical description for a Single Degree of Freedom (SDOF) system is the following:

$$
\left\{\begin{array}{l}
m \dddot{u}+c \dot{u}+F=p \\
F=F^{e l}+F^{p l}=a k u+(1-a) k z \\
\dot{z}=\dot{u}\left[1-\left|\frac{z}{z_{y}}\right|^{n}(\gamma+\beta \operatorname{sgn}(z \dot{u}))\right]
\end{array}\right.
$$

where $F$ is the total Bouc-Wen restoring force, $z$ is the introduced hysteretic parameter i.e. the hysteretic part of displacements or curvatures, $\operatorname{sgn}$ is the signum function and $\alpha$ is the ratio of the post yield stiffness to the initial elastic 
stiffness $k$. The formulation is based on a first order nonlinear differential equation with respect to $z$.

According to Bouc-Wen model, for a Single Degree of Freedom system, the restoring force can be expressed as the sum of an elastic $F^{e l}$ and an inelastic $F^{p l}$ part. Therefore, the model can be visualized as the parallel connection of two springs, one being elastic and the other inelastic.

Regarding the parameters in the Bouc-Wen model $n$ controls the smoothness of the transition from the elastic to the inelastic regime. As $n$ increases the response approaches that of a bilinear model. Parameters $\beta$ and $\gamma$ control the behavior during unloading. In the case where $\beta=\gamma=0.5$ the unloading branches are straight lines parallel to the initial elastic (Figure 2).

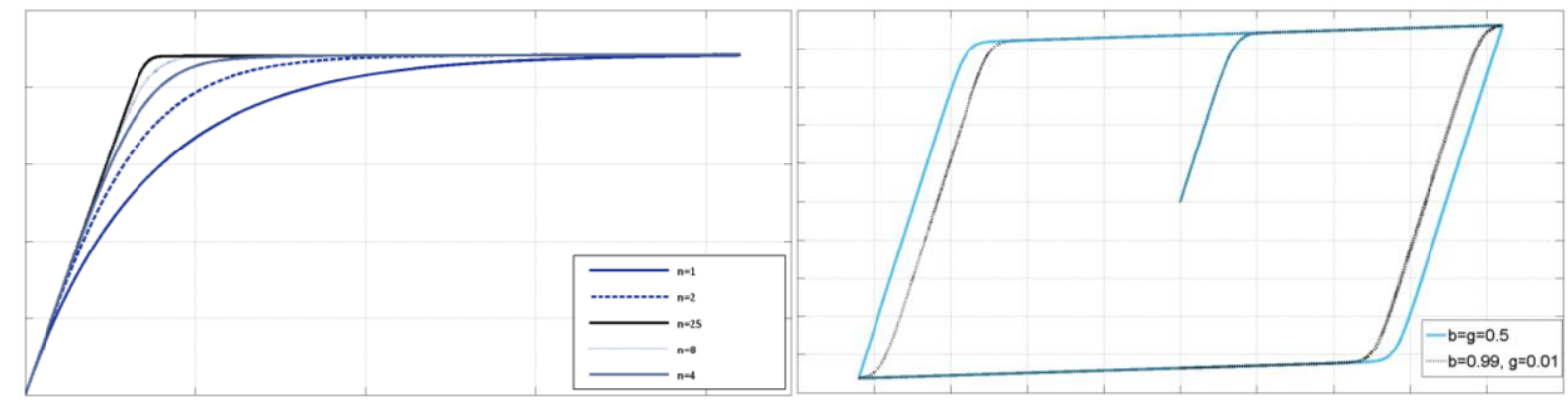

Fig 2. Parameters of the Bouc-Wen hysteretic model.

\section{Implementation of the Bouc-Wen model}

The local springs forces at the middle of the facet are expressed at the computational nodes $F_{x}, F_{y}$ and $M$, through the relation:

$$
\left\{\begin{array}{c}
F_{x}^{1} \\
F_{y}^{1} \\
M^{1} \\
F_{x}^{2} \\
F_{y}^{2} \\
M^{2}
\end{array}\right\}=[B]^{T}\left\{\begin{array}{l}
F_{n} \\
F_{t} \\
M
\end{array}\right\}
$$

According to Bouc-Wen, the spring forces can be decoupled into an elastic and a hysteretic part as:

$$
\begin{gathered}
F_{n}=a k_{n} \delta_{n}+(1-\alpha) k_{n} z_{n} \\
F_{t}=a k_{t} \delta_{t}+(1-\alpha) k_{t} z_{t} \\
M=a k_{\varphi} \varphi+(1-\alpha) k_{\theta} z_{\varphi}
\end{gathered}
$$

or in matrix form:

$$
\left\{\begin{array}{l}
F_{n} \\
F_{t} \\
M
\end{array}\right\}=\left[\begin{array}{ccc}
a k_{n} & 0 & 0 \\
0 & a k_{t} & 0 \\
0 & 0 & a k_{\varphi}
\end{array}\right]\left\{\begin{array}{l}
\delta_{n} \\
\delta_{t} \\
\varphi
\end{array}\right\}+\left[\begin{array}{ccc}
(1-a) k_{n} & 0 & 0 \\
0 & (1-a) k_{t} & 0 \\
0 & 0 & (1-a) k_{\varphi}
\end{array}\right]\left\{\begin{array}{l}
z_{n} \\
z_{t} \\
z_{\varphi}
\end{array}\right\}
$$


Substituting into (13) equations (5) and (12) the following expression can be derived:

$$
\left\{\begin{array}{l}
F_{x}^{1} \\
F_{y}^{1} \\
M^{1} \\
F_{x}^{2} \\
F_{y}^{2} \\
M^{2}
\end{array}\right\}=[B]^{T}\left[\begin{array}{ccc}
a k_{n} & 0 & 0 \\
0 & a k_{t} & 0 \\
0 & 0 & a k_{\varphi}
\end{array}\right][B]\left\{\begin{array}{l}
u_{1} \\
w_{1} \\
\theta_{1} \\
u_{2} \\
w_{2} \\
\theta_{2}
\end{array}\right\}+[\mathrm{B}]^{T}\left[\begin{array}{ccc}
(1-a) k_{n} & 0 & 0 \\
0 & (1-a) k_{t} & 0 \\
0 & 0 & (1-a) k_{\varphi}
\end{array}\right]\left\{\begin{array}{l}
z_{n} \\
z_{t} \\
z_{\varphi}
\end{array}\right\}
$$

where the derivatives of the hysteretic parts with respect to time are following the Bouc-Wen first order differential equations:

$$
\left\{\begin{array}{l}
\dot{z}_{n}=\dot{\delta}_{n}\left[1-\left|\frac{z_{n}}{z_{y}^{n}}\right|^{n}\left(\gamma+\beta \operatorname{sgn}\left(z_{n} \dot{\delta}_{n}\right)\right)\right] \\
\dot{z}_{t}=\dot{\delta}_{t}\left[1-\left|\frac{z_{t}}{z_{y}^{t}}\right|^{n}\left(\gamma+\beta \operatorname{sgn}\left(z_{t} \dot{\delta}_{t}\right)\right)\right] \\
\dot{z}_{\varphi}=\dot{\varphi}\left[1-\left|\frac{z_{\varphi}}{z_{y}^{\varphi}}\right|^{n}\left(\gamma+\beta \operatorname{sgn}\left(z_{\varphi} \dot{\varphi}\right)\right)\right]
\end{array}\right.
$$

The hysteretic matrix can then be defined as [9]:

$$
\left[K_{h}\right]=[B]^{T}\left[\begin{array}{ccc}
(1-a) k_{n} & 0 & 0 \\
0 & (1-a) k_{t} & 0 \\
0 & 0 & (1-a) k_{\varphi}
\end{array}\right]
$$

where parameter $\alpha$ is considered the same for all springs. In general, it can be different and is material dependent and can be identified through simple monotonic loading test. Combining all the elements hysteretic stiffness matrices with the direct stiffness method, the structure hysteretic matrix can be derived, which is orthogonal with dimensions $\left(3 n, 3 n_{e l}\right)$ where $n$ is the number of nodes and $n_{e l}$ is the number of elements. The dynamic equation of motion is stated as:

$$
[M]_{s}\{\ddot{u}\}+[C]_{s}\{\dot{u}\}+[K]_{s}\{u\}+\left[K_{h}\right]_{s}\{z\}=\{P(t)\}
$$

The global equation of motion together with the elemental differential equations form a system that needs to be solved simultaneously. The solution can be achieved by converting the system into state-space form. This is achieved by introducing as auxiliary unknown the vector of nodal velocities. This way the system of equations converts to a set of linear differential equations of first order coupled with the nonlinear Bouc-Wen hysteretic equations (15) as:

$$
\left\{\begin{array}{l}
\{\dot{u}\} \\
\{\ddot{u}\}
\end{array}\right\}=\left[\begin{array}{ccc}
0 & I & 0 \\
-[M]^{-1}[K] & -[M]^{-1}[C] & -[M]^{-1}\left[K_{h}\right]
\end{array}\right]\left\{\begin{array}{l}
\{u\} \\
\{\dot{u}\} \\
\{z\}
\end{array}\right\}+\left\{\begin{array}{c}
0 \\
\{P(t)\}
\end{array}\right\}
$$

with the nonlinear hysteretic equations being in the form of:

$$
\{\dot{z}\}=f(\{\dot{u}\},\{z\})
$$


The above system, for a given dynamic loading and initial conditions can be integrated using robust numerical integrators like Runge-Kutta or Livermore algorithms or variable order solvers based on numerical differentiation formulas which are proved more efficient and are used in this work.

\section{Fracture representation}

Several spring braking rules have been proposed by authors mainly based on critical energy or critical stress. In this work a simpler approach is utilized. Both crack initiation and crack propagation obey simple cut-off rule in combination with a Mohr-Coulomb type of criterion [4].

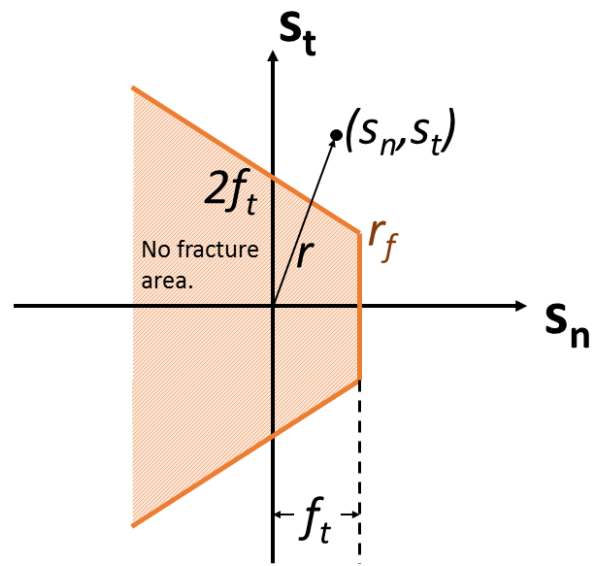

Fig. 3. Mohr-Coulomb type fracture criterion.

In each computational cycle the stresses of the normal $\left(s_{n}\right)$ and tangential $\left(s_{t}\right)$ spring are calculated:

$$
s_{n}=\frac{q_{n}}{\left(\mathrm{~S}_{\mathrm{ij}} t\right)}, s_{t}=\frac{q_{t}}{\left(\mathrm{~S}_{\mathrm{ij}} t\right)}
$$

where $S_{i j}$ is the length of the facet and $t$ is the thickness of the structure and $q_{n}$ and $q_{t}$ the normal and tangential forces acting on the local respective springs. These are then used to calculate $r$ as:

$$
r=\sqrt{\left(\mathrm{s}_{n}^{2}+\mathrm{s}_{t}^{2}\right)}
$$

and are compared to the fracture surface with the criterion:

$$
R=\frac{r}{r_{\mathrm{f}}}>1
$$

where $r_{f}$ designates the area in the stress field, inside which every pair of normal and tangential stresses don't lead to fracture (Figure 3) and is defined using $f t$, the tensile strength of the material. If the fracture criterion is satisfied then for this surface, and therefore element, brittle fracture is assumed. This means that the element spring forces are released and the local spring stiffnesses are set to zero. Only one fracture event is allowed for each computational cycle. In order to advance to the next computational step all stresses must lie within the fracture surface. In addition, the criterion assumes traction free cracks and although, using the Voronoi tessellation the mesh bias regarding crack movement is minimized, crack propagation is not objective with different mesh sizes. Although simple in its apprehension and implementation, the fracture criterion should be extended to account for proper fracture energy calculation. 


\section{Numerical examples}

\subsection{Simply supported beam}

In this example a simply supported beam with a load at its middle is examined. Two different discretization schemes are employed using 275 and 689 nodes. Material is elastic, concrete C20/25 with Young's modulus E=29 Gpa, Poisson ratio $v=0.2$ and the dimensions of the beam are $1 \times 0.2 \mathrm{~m}$. The final configuration of the deformed beam together with the developed cracks are presented in Figure 4. The model is able to predict the correct form and shape of the cracks.
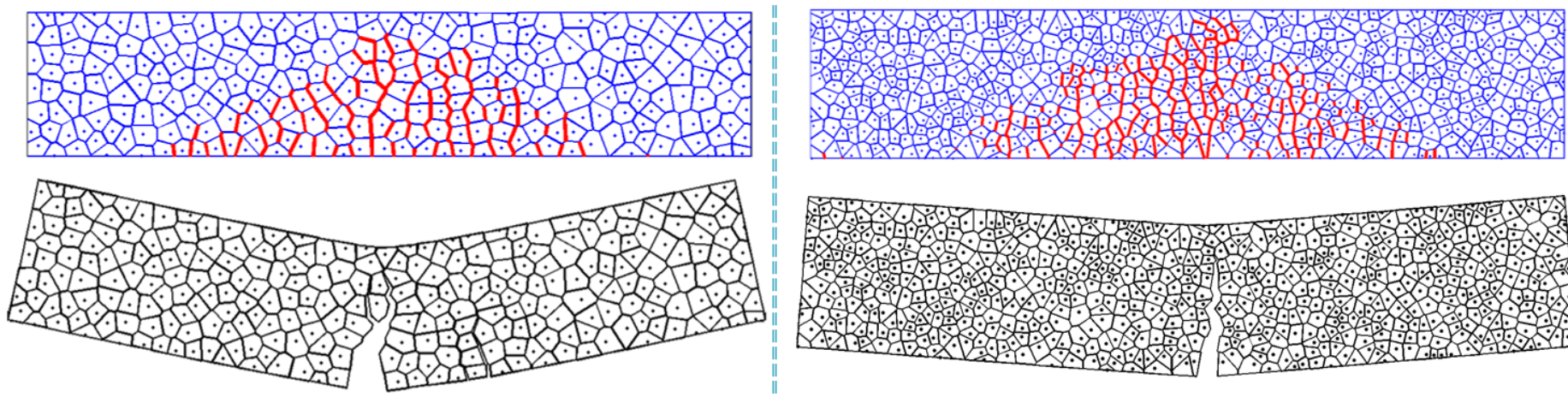

Fig. 4. Crack patterns in simply supported beam.

\subsection{Cantilever beam}

For this second example a cantilever beam with a concentrated load at the free end is examined. The beam geometry together with the discretization can be seen in Figure 5. For this example, the beam was discretized with 94 nodes producing 219 elements.

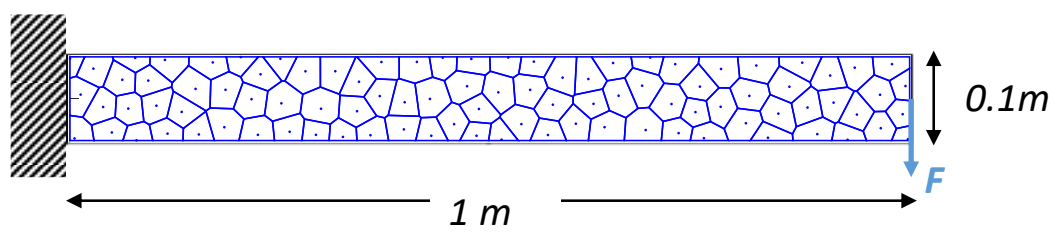

Fig. 5. Beam geometry and discretization.

Material is steel with $\sigma_{y}=240 \mathrm{MPa}$ and Young's modulus is $E=210 \mathrm{Gpa}$ while Poisson ratio is $v=0.3$. Beam thickness is $0.05 \mathrm{~m}$ and the Bouc-Wen parameters are $n=8$ and $\alpha=\beta=0.5$ while the ratio of the post yielding branch to the initial elastic one is set to $2 \%$ hardening. Since the beam has a span to depth ratio larger than 3 , the beam yield load and ultimate load can be calculated analytically:

$$
P_{y}=\frac{\sigma_{y} b h^{2}}{6 L}=20 k N ; \quad P_{u}=\frac{\sigma_{y} b h^{2}}{4 L}=30 k N
$$




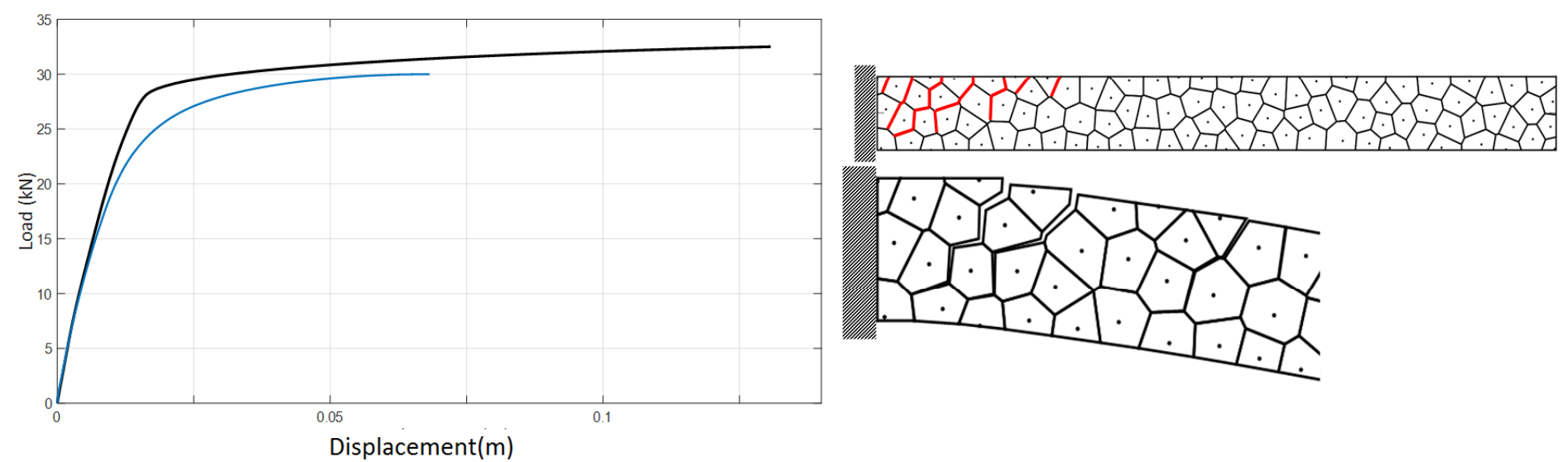

Fig. 6. External load-tip displacement diagram with and without fracture and magnified crack pattern.

In Figure 6, results obtained from two analyses are presented. In the first analysis only plasticity was considered for the internal springs and no fracture was involved (black line). In the second analysis fracture was also taken into account (blue line). Results show that after initial yield some elements near the support rupture, resulting in a gradual stiffness loss until the external force reached around $30 \mathrm{kN}$ when most of the cross section has lost its stiffness and essentially developed a hinge. In Figure 6 the crack patterns developed in the beam are also presented. The red lines denote the facets in which the springs have ruptured. Also in the same figure the base of the beam near the support is presented magnified in order to show the main cracks developed and their propagation through the beam.

\section{Conclusions}

In this work the concept of the Rigid Body Spring Network model has been extended to account for hysteretic and fracture phenomena. Material is discretized based on a Voronoi tessellation into convex polygons, which are the cells that form the discrete rigid bodies of the model. In the middle of their common facets lie three zero length springs that undergo all the deformations of the structure.

The behavior of springs follows the smooth hysteretic Bouc-Wen model. The parameters and behavior of this model are explained and its implementation in the RBSN framework has been presented. Random mesh generation, using only a minimum distance criterion, effectively minimizes the mesh bias towards crack propagation due to monotonic loading and ensures the elastic uniformity. Crack initiation and propagation obey simple cut-off and Mohr-Coulomb type of criterion. This gives the ability to identify regions where plasticity and fracture are developed. However, the fracture model should be extended to account for the calculation of the fracture energy.

Based on this formulation, under monotonic loading, plastic regions and fracture events can be closely identified and monitored for the overall structure. Numerical results are presented that validate the proposed method.

\section{Acknowledgements}

The authors would like to acknowledge the support from the "RESEARCH PROJECTS FOR EXCELLENCE IKY/SIEMENS".

\section{References}

[1] T. Kawai, "New discrete models and their application to seismic response analysis of structures," Nuclear Engng. Design., vol. 48, pp. 207-229, 1978.

[2] J. E. Bolander, G. S. Hong, "Rigid-Body-Spring Network modeling of prestressed concrete members," ACI Structural Journal, vol. 99, no. 5, pp. 595-604, 2002.

[3] J. E. Bolander, K. Moriizumi, M. Kunieda, M. Yip, "Rigid-Body-Spring Network modeling of cement-based composites," Fracture Mechanics of Concrete Structures, pp. 773-780, 2001.

[4] J. E. Bolander, S. Saito, "Fracture analyses using spring networks with random geometry," Eng Fract Mech., vol. 61, no. 5-6, pp. 569-591, 1998. 
[5] K. Kim, Y. M. Lim, "Simulation of rate dependent fracture in concrete using an irregular lattice model," Cement and Concrete Composites, vol. 33, no. 9, pp. 949 - 955, 2011.

[6] F. P. Preparata, M. I. Shamos. Computational Geometry-An introduction. Springer, New York, 1985.

[7] R. Bouc, "Forced vibration of mechanical system with hysteresis," in Proceedings of 4th Conf. on Nonlinear Oscillation, Prague, 1967.

[8] Y. K. Wen, "Equivalent linearization for hysteretic system under random excitation," Journal of Applied Mechanics, vol. 47, no. 1, pp. 150-154, 1980.

[9] S. D. Christos, K. K. Vlasis, "Plane stress problems using hysteretic rigid body spring network models," Comp. Part. Mech. doi:10.1007/s40571-016-0128-1, 2016. 